\title{
THEORETICAL SEX RATIOS OF DIOECIOUS AND GYNODIOECIOUS ANGIOSPERMS
}

\author{
DAVID G. LLOYD \\ Botany Department, University of Canterbury, Christchurch, \\ New Zealand
}

Received 3.i.73

\section{SUMmaRY}

The following parameters affecting the frequency of male and female plants in sexually dimorphic Angiosperms are defined: relative fecundity (as seed parent), $F$, differential survival, $S$, and gamete success, $G$, all as male/female quotients, and the probability of fertilisation of ovules on female plants, in terms of the number of pollinator visits to the flowers, $x$.

Four different modes of inheritance of gynodioecy and dioecy are examined:

(a) Heterozygous female model-female $\mathrm{Mm}$, male $\mathrm{mm}$.

(b) Dual male model-female $m m$, male $M M$ and $M m$.

(c) Heterozygous male model-female $m m$, male $M m$ only.

(d) Cytoplasmic inheritance.

The equilibrium frequencies of females and (for three models) the rates of change in female frequency per generation at non-equilibrium frequencies are expressed in terms of the controlling parameters.

The conditions necessary for sexual dimorphism and the changes in frequencies of the sex genotypes as the number of pollinator visits increase and the relative seed fecundity decreases towards zero (dioecy) are derived for each model.

Differential survival of the sexes (and differential gamete success in the heterozygous male model) cause an increase at equilibrium in the sex surviving more frequently or the sex derived from the more successful gamete. In a dioecious population, the ratio of the sexes is equal to their relative survival or their relative gamete success.

Populations at non-equilibrium frequencies return more rapidly to equilibrium with the heterozygous male model than with the heterozygous female and cytoplasmic models. In the extreme case when females are lost from a population, with the heterozygous female and cytoplasmic models extinction or loss of sexual dimorphism occurs, but with the heterozygous male and dual male models sexual dimorphism is regenerated. The ability of systems with heterozygous males to regenerate dimorphism may explain the prevalence of such systems in both gynodioecious and dioecious populations.

\section{InTRODUCTION}

THE quantitative effects of various parameters on the sex ratios of dioecious and gynodioecious Angiosperms have been examined by several authors. Lewis (1941) calculated the equilibrium proportions, $p$, of male-sterile (female) plants with different types of inheritance. He assumed that the fitness of females was a function of their seed fertility relative to hermaphrodites, $f$, and a term $(1-p)^{x}$ which allows for the effect of the proportion of hermaphrodites, $1-p$, on the efficiency of pollination. Hermaphrodites were assumed to have a seed fertility of 1 and supply all the pollen of the population. For both dominant and recessive female genes, Lewis found that at equilibrium,

$$
p=\frac{1}{2}-\frac{1}{f(1-p)^{x-1}}
$$


From this equation, females are eliminated unless their fertility is more than twice that of hermaphrodites. As the relative fertility of females increases further, their expected frequency rises towards one-half at a rate dependent on the value of $x$. No precise model for the solution of $x$ was offered, but Lewis considered that its true value probably lies between 0.5 and 2 . When the female phenotype is determined cytoplasmically, Lewis (1941) calculated that females are present at equilibrium if they have only a slight advantage over hermaphrodites in seed fertility and that the proportion of females approaches one as $f$ moves towards infinity (dioecy). Using numerical examples, Jain (1961) calculated that male-sterile plants controlled by a recessive gene are retained at intermediate frequencies in a hermaphrodite population if there is considerable overdominance at the male-sterility locus and hermaphrodites are frequently inbred. Ross and Shaw (1971) used a computer simulation model to study the effect of the relative seed fertility of the sexes on the frequencies of females when sex is determined by a pair of unlinked loci with three different modes of gene interaction. After 500 generations, the frequencies of female plants with all three modes of inheritance are virtually identical to the frequencies predicted with Lewis' monogenic models.

A number of deterministic, discrete generation models presented below study the effects on sex ratios of the relative seed contribution of the sexes, precise levels of pollination for animal-pollinated species, sex-differential mortality and (where appropriate) gamete selection among pollen nuclei. The equilibrium proportions of the sexes in terms of these parameters are obtained for three single gene models and cytoplasmic inheritance. Expressions for the rate of change in female frequency at non-equilibrium proportions are also obtained for most models.

\section{Parameters USED IN THE MODELS}

\section{(i) Sex proportions}

The proportion of females in a population is $p$ and that of males $1-p$. The rate of change in female frequency per generation is $\Delta p$.

\section{(ii) Quantification of sex expression}

Dioecious and gynodioecious populations are considered here. Definitions of these systems usually state that dioecious populations consist of male and female plants producing male and female gametes respectively, while gynodioecious populations contain hermaphrodites producing male and female gametes as well as females producing only female gametes. The distinction between the two classes of sex expression is, however, not as clear as the definitions imply. In many dioecious populations the plants are not always strictly unisexual, but bear a proportion of flowers with gametes of the opposite sex. This inconstancy is usually more frequent among males than among females (Allen, 1940). A subsequent paper will survey data showing that the seed production of polleniferous plants of gynodioecious species is typically considerably less than that produced by females, but varies continuously from approximately equal to the seed production of females to very low quantities approaching strict dioecy, i.e. gynodioecy merges into dioecy. It will also be calculated there that the polleniferous plants of gynodioecious populations contribute their genes to the next generation 
predominantly via pollen and the genetic contribution via ovules is less (much less if females are common). Consequently, the polleniferous plants of gynodioecious populations, as well as those of dioecious populations, will be described below as males (cf. Lloyd, 1972, 1973a). Thus in the models considered below the males of gynodioecious populations contribute a minor but appreciable proportion of their genes via ovules, whereas the males of dioecious populations produce no seed. It is assumed that in both dioecious and gynodioecious populations females produce no pollen.

Dioecy and gynodioecy can be considered together by the use of a parameter, the relative seed feduncity, $F$, which measures the maternal components of the comparative seed contributions of males and females. The overall seed contributions which male and female individuals make to the gene pool of the next generation at reproduction are determined by a number of factors:

1. Characteristics of the two sexes as seed parents. The relative number and subsequent fate of seeds produced by males and females is influenced by the number of ovules they produce, their comparative quality, the magnitude of heterosis and the percentage of self-fertilisation of the ovules borne on male plants. Males may have lower seed contributions than females because they produce fewer or poorer seeds or because a proportion of their seeds result from self-fertilisation.

2. The probabilities of fertilisation of the ovules produced on males and females, controlled by pollination conditions and any barriers to the selffertilisation of males.

3. Differential mortality between male and female offspring due to their own sex genotypes.

4. The sex proportions among the seed progeny of males and females, controlled by the mode of sex inheritance and, where the males are heterozygous for sex-determining alleles, by the gametic success of male- and femaledetermining pollen nuclei.

The factors included under 2, 3 and 4 act in different ways and are treated separately below. The maternal components of the seed contributions of the sexes included under 1 cannot be readily separated from each other and contribute jointly to the relative seed fecundity of the sexes. This can be estimated with varying degrees of accuracy from the relative seed numbers or seed weights of the sexes (Lloyd, unpublished). The measure used here, the relative (male/female) seed fecundity, $F$, has a more convenient scale, from zero to one, than the inverse measure for female fertility, $f$, employed by Lewis (1941) and Ross and Shaw (1971). The latter varies from one to infinity (ignoring the unimportant cases when females contribute fewer offspring via seeds than males and are invariably eliminated). In the families of curves (figs. 1-7) showing the effects of the relative fecundity on equilibrium sex ratios for particular modes of inheritance and values of other parameters, the scale for the relative fecundity decreases from one on the left to zero on the right. In this way, the changes in sex ratio that a gynodioecious population $(1 \geqq F>0$ ) would undergo if the seed contribution of males was gradually reduced towards zero (dioecy) are conveniently shown. The scale used does not presume, however, that gynodioecy necessarily evolves to dioecy or even in that direction or that dioecy always evolves from gynodioecy. 


\section{(iii) Pollination levels}

The effects of cross- and self-fertilisation of males are included in the value of the relative male fecundity, as previously discussed, so the frequency of self-fertilisation is not considered separately. It is assumed that all functional ovules of male plants are fertilised, either by self- or cross-pollination. The probability of fertilisation of the ovules of female plants can be expressed exactly in terms of the sex proportions and the number of visits to each flower of female plants by the following model for animal-pollinated populations. Consider that during a foraging trip, a pollinator makes a number of visits to flowers of male and female plants at random and that a single visit to a flower of a female plant results in fertilisation of all ovules if the immediately preceding visit was to a flower of a male plant. When a flower of a female plants is visited $1,2,3, \ldots, x$ times, the probabilities that all immediately preceding visits were to females are $p, p^{2}, p^{3}, \ldots, p^{x}$. Hence the fractions of ovules of female plants fertilised if each of their flowers is visited $1,2,3, \ldots, x$ times are the probabilities that at least one of the immediately preceding visits to each flower was to a flower of a male plant, i.e. $1-p, 1-p^{2}, 1-p^{3}$, $\ldots, 1-p^{x}$. If the pollinator activity is less than one visit to every female flower, it is assumed that a fraction $x^{\prime}$ of these flowers is visited once only and that the remainder are not visited. Then the fraction of fertilised ovules is $x^{\prime}(1-p)$. The pollination levels for which sex proportions are calculated below range from $x^{\prime}=0 \cdot 25$ to $x=\infty$ (probability of fertilisation $=1$ ). The latter level is mathematically equivalent to the more general situation when the probabilities of fertilisation of ovules on male and female plants are equal but less than one, e.g. at any pollination level, if self-fertilisation is completely prevented, as when the males are fully self-incompatible.

\section{(iv) Sex-differential survival}

Differential survival of the sexes which is due to their own sex genotypes is measured by the relative male survival, $S$, defined as the rate of survival from zygote formation to reproductive maturity of males divided by the corresponding survival rate of females.

\section{(v) Gamete selection}

If males are heterozygous for sex-determining genes, selective fertilisation may influence the sex frequencies. The relative gamete success, $G$, is defined as the number of male-determining pollen nuclei achieving fertilisation divided by the corresponding number of female-determining nuclei.

\section{Heterozygous female model}

$$
(\text { Female }=M m, \text { male }=m m)
$$

The simplest genetic model of dioecy and gynodioecy assumes that the female phenotype is due to a dominant allele, $M$. Since females produce no pollen, no homozygous $M M$ individuals are formed and there are only two genotypes and two kinds of mating to be considered, i.e.

$$
\text { Total seed set }=\left(q \times \sigma^{\star}\right)+(\hat{\sigma} \times \hat{\sigma}) \text {. }
$$

If every flower of female plants is visited one of more $(x)$ times, the probability of fertilisation of the ovules on female plants is $1-p^{x}$, i.e.

$$
\text { Total seed set }=p\left(1-p^{x}\right)+(1-p) F \text {. }
$$


Female $\times$ male matings produce half female $(M m)$ offspring with a relative survival of 1 and half male $(\mathrm{mm})$ offspring with relative survival, $S$, while male $\times$ male matings produce only male $(\mathrm{mm})$ offspring with relative survival,

$S$. Therefore,

Composition of mature offspring

$$
=p\left(1-p^{x}\right)\left(\frac{1}{2} M m+\frac{S}{2} m m\right)+(1-p) F S m m .
$$

Total offspring $=p\left(1-p^{x}\right)\left(\frac{1+S}{2}\right)+(1-p) F S$.

Frequency of female offspring

$$
=\frac{p\left(1-p^{x}\right)}{2} /\left[\frac{p\left(1-p^{x}\right)(1+S)}{2}+(1-p) F S\right] \text {. }
$$

The change in female frequency in one generation,

$$
\begin{aligned}
\Delta p & =(\text { frequency female offspring })-p \\
& =\frac{p\left(1-p^{x}\right)(1-p-S p)-2 p(1-p) F S}{p\left(1-p^{x}\right)(1+S)+(1-p) F S}
\end{aligned}
$$

At equilibrium, $\Delta p=0$, i.e. $p=0$ or

$$
\left(1-p^{x}\right)(1-p-S p)-2(1-p) F S=0 .
$$

If only a fraction $\left(x^{\prime}\right)$ of the flowers are visited, the probability of fertilisation of the ovules of females is $x^{\prime}(1-p)$. Then,

Composition of mature offspring

$$
=(1-p)\left[\frac{p x^{\prime}}{2} M m+\left(\frac{p x^{\prime} S}{2}+F S\right) m m\right]
$$

Total offspring $\quad=(1-p)\left[\frac{p x^{\prime}(1+S)}{2}+F S\right]$.

Frequency of female offspring

$$
\begin{gathered}
=\frac{p x^{\prime}}{p x^{\prime}+p x^{\prime} S+2 F S} . \\
\Delta p=\frac{p\left(x^{\prime}-p x^{\prime}-p x^{\prime} S-2 F S\right)}{p x^{\prime}+p x^{\prime} S+2 F S} .
\end{gathered}
$$

At equilibrium, $\Delta p=0$, i.e. either $p=0$ or

i.e.

$$
x^{\prime}-p x^{\prime}-p x^{\prime} S-2 F S=0
$$

$$
p=\frac{x^{\prime}-2 F S}{x^{\prime}(1+S)}
$$

Formulae for the equilibrium female frequency in terms of the relative seed fecundity, obtained from equations 2 and 4 for chosen values of $x$ (and $x^{\prime}$ ) and $S$ are given in table 1 . Numerical values of $p$ obtained from these formulae for a selected set of values of $F$ are also shown in table 1 and graphed against the $F$ values to give the families of curves in figs. 1 and 2 . 


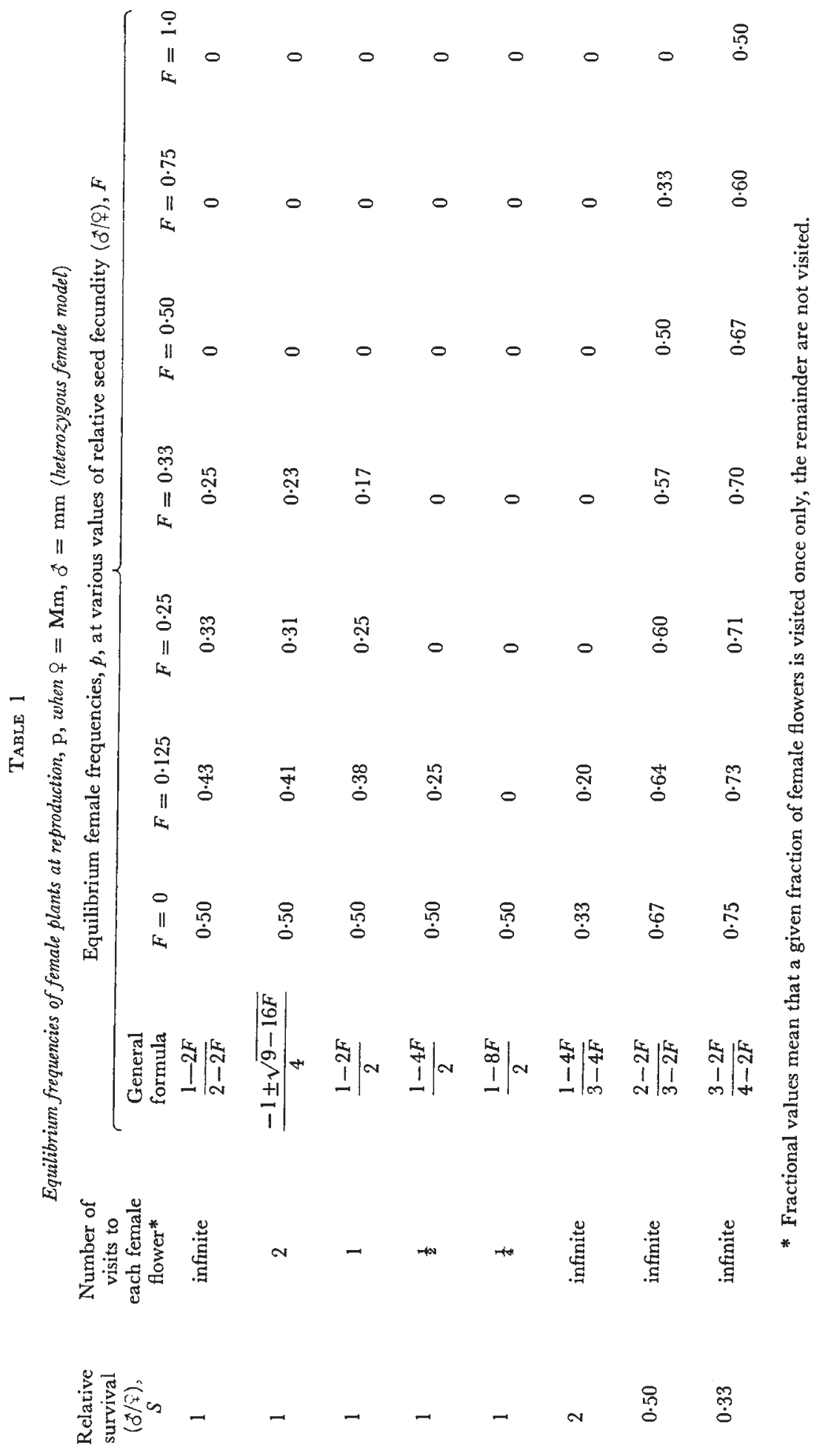


When there is no sex-differential survival $(S=1)$ and there are one or more visits to each flower, the equilibrium female frequencies obtained agree with the principal results of Lewis (1941), Valdeyron (1967) and Ross and Shaw (1971). That is, no females are present in the equilibrium population unless the male seed fecundity is less than half that of females $(F<0.5)$, and

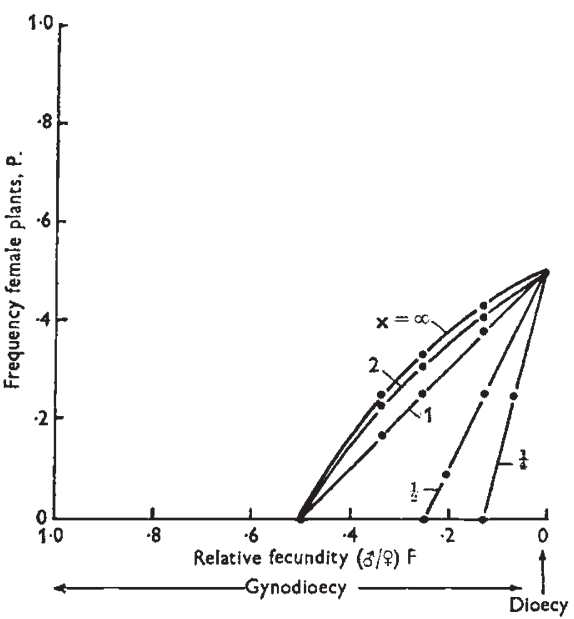

FIG. 1

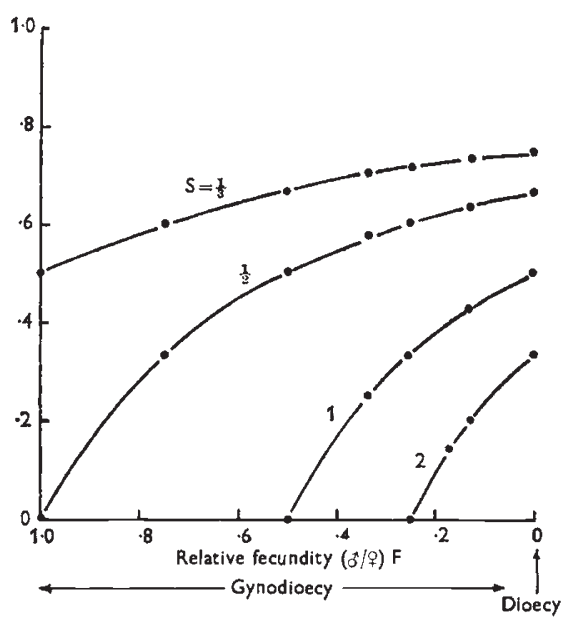

Fig. 2

FIGs. 1 and 2.-Heterozygous female model-relationships between the relative seed fecundity, $F$, and the equilibrium frequency of female plants, $p$, at various values of relative survival, $S$, and number of pollinator visits, $x$. Fig. 1.-Female frequency curves for different numbers of pollinator visits (shown beside each curve), when there is no differential survival $(S=1)$. Fig. 2.-Female frequency curves for different values of relative survival (shown beside each curve), when the probabilities of fertilisation of ovules on male and female plants are equal $(x=\infty)$.

the frequency of females rises to a maximum of 0.5 as $F$ falls to 0 (dioecy) (fig. 1). At values of $F$ between 0.5 and 0 , the female frequency at equilibrium is dependent on the pollination level and increases slightly as the number of pollinator visits increases. If only a fraction of flowers is visited, the pollination level has a greater effect. No females are present at equilibrium unless the relative seed fecundity falls below a value less than 0.5 which depends on the fraction of flowers visited, but the expected frequency of females still rises to 0.5 in a dioecious population.

If the sexes differ in their survival rates, both the maximum value of $F$ permitting sexual dimorphism and the female frequency in dioecious populations differ from 0.5 (fig. 2). In both dioecious and gynodioecious populations, the sex with better survival has a higher equilibrium frequency then when males and females survive at the same rate. In dioecious populations $(F=0)$, from equations 2 and 4 ,

$$
p=\frac{1}{1+S}
$$

or

$$
\frac{1-p}{p}=S
$$


That is, regardless of the pollination level, the ratio of the sexes at equilibrium in a dioecious population is equal to their relative survival rates.

The expression for $\Delta p$, obtained from either equation 1 or equation 3 , when all flowers are visited once only $\left(x\right.$ or $\left.x^{\prime}=1\right)$ and there is no difference between the sexes in survival rates is shown in table $5 \mathrm{~A}$. Numerical values. of $\Delta p$ for some particular values of $F$ and some non-equilibrium female frequencies are also shown and compared with the difference between the chosen non-equilibrium frequencies and the equilibrium frequency. In a dioecious system, a non-equilibrium population with females present $(p>0)$ returns to the equilibrium position in a single generation. A gynodioecious population which is not at equilibrium, however, returns only part of the distance (usually 20 to 60 per cent.) to equilibrium in a single generation. If females are by chance lost entirely $(p=0)$ from either dioecious or gynodioecious populations, they are not regained $(\Delta p=0)$, except by mutation or gene flow from another population.

\section{DuAl male model}

$$
(\text { Female }=m m \text {, males }=M M \text { and } M m)
$$

Lewis (1941) examined the equilibrium frequency of female plants determined by a recessive gene, with general, unspecified pollination levels and in the absence of sex-differential mortality and gamete selection. The equation he obtained was the same as that for a dominant female gene (the heterozygous female model treated above). This "dual male" genetic model is reconsidered here, and separate equilibrium frequencies for the two male genotypes are derived for the first time. The occurrence of two male genotypes complicates the treatment considerably, and equilibrium frequencies are obtained only for the simplest situation-the absence of sexdifferential mortality and gamete selection $(S, G=1)$ and equal probabilities of fertilisation for ovules on male and female plants. Even then, it is necessary to adopt a simpler method of derivation, similar to Lewis' method, which assumes that at equilibrium the proportions of the three genotypes are identical in successive generations.

The frequency of females $(\mathrm{mm})$ is taken as $p$, that of $M m$ males as $q$ and that of $M M$ males as $r$. The two male genotypes are assumed to have the same relative seed fecundity, $F$, and produce the same quantity of pollen. So $M m$ and $M M$ males produce the fractions $q /(q+r)$ and $r /(q+r)$ of the pollen produced, respectively. For simplicity, the males are assumed to be entirely self-pollinated. Then,

Total seed set

$$
=(m m \times M m)+(m m \times M M)+(M m \otimes)+(M M \otimes) .
$$

Composition of offspring

$$
\begin{aligned}
& =\frac{p q}{q+r}\left(\frac{1}{2} M m+\frac{1}{2} m m\right)+\frac{p r}{q+r}(M m)+q F\left(\frac{1}{4} M M+\frac{1}{2} M m+\frac{1}{4} m m\right)+r F(M M) \\
& =\left[\frac{p q}{2(q+r)}+\frac{F q}{4}\right] m m+\left[\frac{p q}{2(q+r)}+\frac{p r}{q+r}+\frac{F q}{2}\right] M m+\left[\frac{F q}{4}+F r\right] M M \\
& =p_{1}+q_{1}+r_{1},
\end{aligned}
$$


where $p_{1}, q_{1}$ and $r_{1}$ are the proportions of the genotypes among the offspring. If these proportions are the same as those of the parents, $p_{1}=k p, q_{1}=k q$ and $r_{1}=k r$, where $k$ is an unknown constant. That is,

and

$$
\begin{gathered}
\frac{p q}{2(q+r)}+\frac{F q}{4}=k p, \\
\frac{p q}{2(q+r)}+\frac{p r}{q+r}+\frac{F q}{2}=k q,
\end{gathered}
$$

$$
\frac{F q}{4}+F r=k r
$$

Adding equations 7, 8 and 9 and substituting $1-p$ for $q+r$,

Adding 8 and $9 \times 2$,

$$
p+F(1-p)=k \text {. }
$$

From 10 and 11 ,

$$
\frac{p}{2(1-p)}+F=k
$$

$$
p+F(1-p)=\frac{p}{2(1-p)}+F
$$

i.e. frequency of $m m$ females,

$$
p=\frac{1-2 F}{2-2 F} .
$$

Substituting the expression for $p$ from 12 in 10 gives $k=\frac{1}{2}$. Substituting for $p$ and $k$ in 7 and putting $q+r=1-p$, frequency of $M m$ males,

$$
q=\frac{1-2 F}{(1-F)(2-3 F)} .
$$

Substituting for $q$ and $k$ in 9, frequency of $M M$ males,

$$
r=\frac{F}{2(1-F)(2-3 F)} .
$$

Equation 12 for the equilibrium frequency of females is the same as that for the heterozygous female model when $S=1$ and the probabilities of fertilisation for ovules on male and female plants are equal (table 1). That is, there is no sexual dimorphism unless $F$ is below 0.5 , and as $F$ is reduced further the equilibrium female frequency rises to 0.5 at dioecy (table 2, fig. 3). The result is equivalent to that of Lewis (1941).

Heterozygous $(\mathrm{Mm})$ males also do not persist in a gynodioecious population if the relative seed fecundity is 0.5 or greater. As $F$ decreases below 0.5 , the frequency of heterozygotes increases rapidly to a maximum value of 0.536 at $F=0.211$, then drops slightly to exactly 0.5 in a dioecious population (table 2, fig. 3 ). The homozygote $(M M)$, in contrast, is the only genotype in an equilibrium population if $F \geqq 0 \cdot 5$. As the relative seed fecundity falls below $0 \cdot 5$, the frequency of male homozygotes declines rapidly to zero when the males contribute no seed. Thus a dioecious population behaves as one with conventional male heterogamety, with no YY males although these are as viable and fertile as $\mathrm{XY}$ males. 


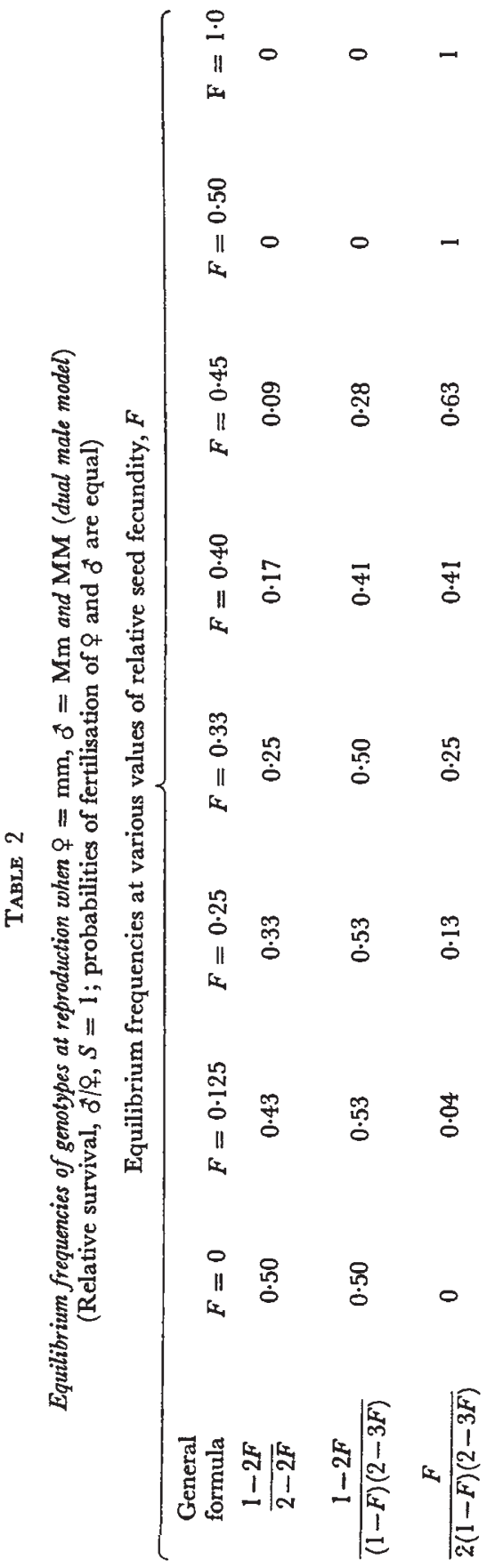

$$
\begin{aligned}
& \text { 总 क त्ञ हु }
\end{aligned}
$$


Expressions for the rates of change of the genotype frequencies from nonequilibrium frequencies have not been obtained for this model. Nevertheless, it is apparent that if females are entirely lost from a population by chance, sexual dimorphism can be regenerated from the seed produced by heterozygous males, in contrast to the heterozygous female model previously discussed.

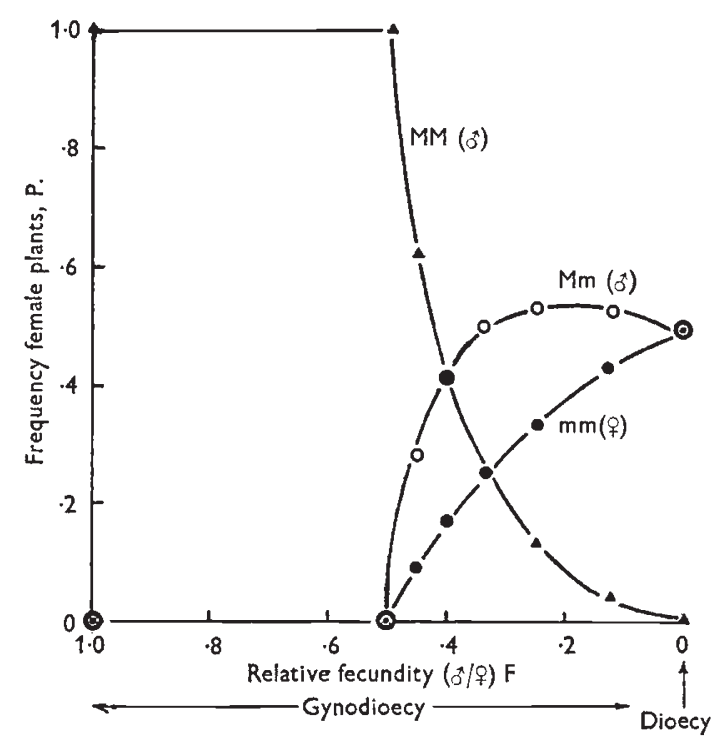

Frg. 3.-Dual male model-the equilibrium frequencies of the three sex genotypes at various values of relative seed fecundity, $F$, when the probabilities of fertilisation of ovules on male and female plants are equal.

\section{Heterozygous male model}

$$
\text { (Female }=m m \text {, male }=M m \text { only) }
$$

This model assumes that the female phenotype is due to a recessive allele, $m$, and the dominant homozygote $(M M)$ is lethal, so all males have the heterozygous $(M m)$ genotype. The sex proportions with this model are derived by the same method as that used for the heterozygous female model, with the addition of another parameter, the relative $(M / m)$ gamete success, $G$, which allows the effect of selective fertilisation among pollen nuclei to be considered. Male $(\mathrm{Mm})$ and female $(\mathrm{mm})$ offspring are produced in the ratio $S G: 1$ following crosses between males and females and in the ratio $S(G+1) / 2: 1 / 2$ following male $\times$ male matings.

If every flower is visited one or more $(x)$ times, the probability of fertilisation of ovules on female plants is $1-p^{x}$. Then,

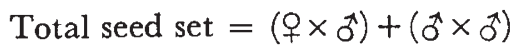

$$
=p\left(1-p^{x}\right)+F(1-p) \text {. }
$$

Composition of offspring

$$
=p\left(1-p^{x}\right)(S G M m+1 m m)+F(1-p)\left[\frac{S(G+1)}{2} M m+\frac{1}{2} m m\right] \text {. }
$$


Total offspring

$$
=(S G+1)\left(1-p^{x}\right) p+\frac{1}{2} F(S G+S+1)(1-p) .
$$

Frequency of female offspring

$$
=\frac{2 p\left(1-p^{x}\right)+F(1-p)}{2(S G+1)\left(1-p^{x}\right) p+F(S G+S+1)(1-p)}
$$

$\Delta p=$ (frequency of female offspring) $-p$

$$
=\frac{2 p\left(1-p^{x}\right)+F(1-p)-2(S G+1)\left(1-p^{x}\right) p^{2}-F(S G+S+1)(1-p) p}{2(S G+1)\left(1-p^{x}\right) p+F(S G+S+1)(1-p)} .
$$

At equilibrium, $\Delta p=0$, i.e.

$$
2 p\left(1-p^{x}\right)+F(1-p)-2(S G+1)\left(1-p^{x}\right) p^{2}-F(S G+S+1)(1-p) p=0
$$

If only a fraction $\left(x^{\prime}\right)$ of flowers are visited, the probability of fertilisation of ovules on female plants is $x^{\prime}(1-p)$. Then,

Composition of offspring

$$
\left.=x^{\prime} p(1-p)(S G M m+1 m m)+F(1-p)\left[\frac{(S G+S)}{2} M m+\frac{1}{2} m m\right)\right] \text {. }
$$

Total offspring

$$
=x^{\prime} p(1-p)(S G+1)+\frac{F}{2}(S G+S+1)(1-p) .
$$

Frequency of female offspring

$$
\begin{aligned}
& =\frac{2 x^{\prime} p(1-p)+F(1-p)}{2 x^{\prime}(S G+1)(1-p) p+F(S G+S+1)(1-p)} \\
& =\frac{2 x^{\prime} p+F}{2 x^{\prime}(S G+1) p+F(S G+S+1)} \\
\Delta p & =\frac{2 x^{\prime} p+F-2 x^{\prime}(S G+1) p^{2}-F(S G+S+1) p}{2 x^{\prime}(S G+1) p+F(S G+S+1)} .
\end{aligned}
$$

At equilibrium, $\Delta p=0$, i.e.

$$
2 x^{\prime}(S G+1) p^{2}+\left(F S G+F S+F-2 x^{\prime}\right) p-F=0 .
$$

The equilibrium female frequencies can be obtained for any particular values of $F, S, G$ and $x$ (or $x^{\prime}$ ) by solution of equation 16 or 18 . The values in table 3 and fig. 4 of female frequencies in the absence of differential mortality and gamete selection $(S, G=1)$ indicate that the heterozygous male model behaves differently from the two previous models. If the inheritance of gynodioecy follows the heterozygous male model, females are frequent at all values of relative seed fecundity. Even when $F=1$, $p=0.35-0.41$, depending on the pollination level. As $F$ decreases, the equilibrium female frequency increases only slightly to a maximum of 0.5 in a dioecious population; the effect of lower values of male fecundity is largely offset by the concomitant decrease in inviable $M M$ homozygotes formed on the males.

The effect of differences between the sexes in the rate of survival on this genetic model are similar to those on the heterozygous female model-the equilibrium frequency of the sex surviving more often is considerably 


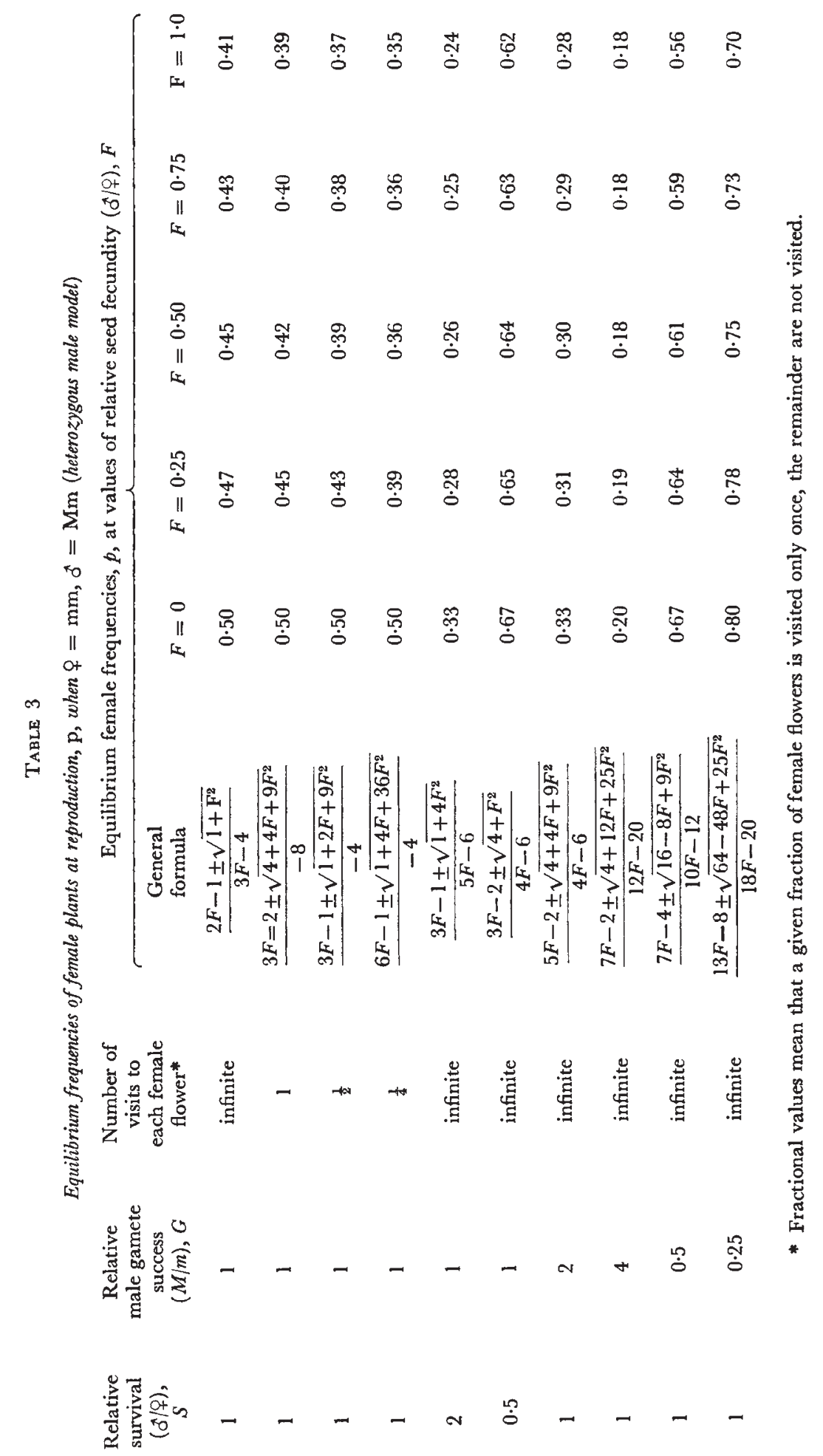


increased (table 3, fig. 5). If males survive twice as frequently as females and male seed fecundity is equal to that of females $(S=2, F=1)$, females still represent almost a quarter of the population.

Gamete selection alters the number of viable seed produced by males as well as the sex proportions among the progeny of both sexes. Hence the effect of gamete selection (table 3, fig. 5) is similar but not identical to that of differential survival, which does not alter the number of seeds produced

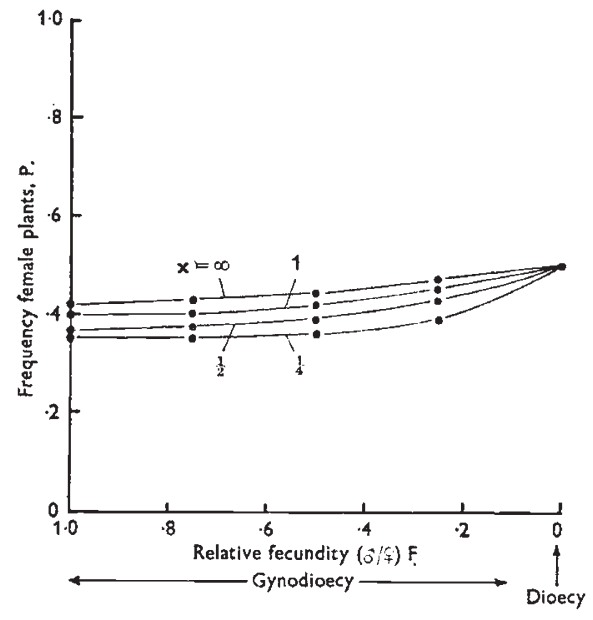

FIG. 4

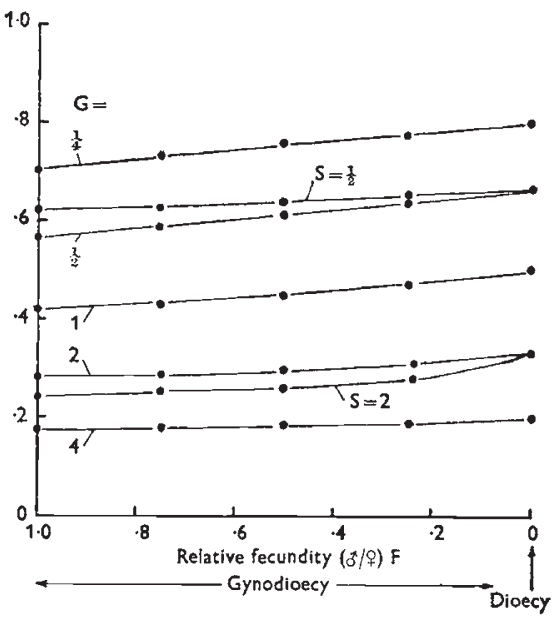

FIG. 5

Figs. 4 and 5.-Heterozygous male model-relationships between the relative seed fecundity, $F$, and the equilibrium frequency of female plants, $p$, with various numbers of pollinator visits, $x$, and values of relative survival, $S$, and differential gamete success, $G$. Fig. 4.Female frequency curves for different numbers of pollinator visits (shown beside each curve), when there is no differential survival $(S=1)$. Fig. 5.-Female frequency curves for different values of relative survival, $S=\frac{1}{2}, 2$, and differential gamete success, $G=\frac{1}{4}, \frac{1}{2}, 1,2,4$, (shown beside each curve), when the probabilities of fertilisation of ovules on male and female plants are equal $(x=\infty)$.

by males. In dioecious populations, however, the two parameters have the same effect. If $F=0$, equation 16 reduces to $2 p\left(1-p^{x}\right)(1-p-S G p)=0$ and equation 18 reduces to $2 p x^{\prime}(S G p+p-1)=0$. In both cases, either $p=0$ or

If $G=1$,

$$
p=\frac{1}{1+S G}
$$

i.e.

If $S=1$,

$$
p=\frac{1}{1+S}
$$

$$
\frac{1-p}{p}=S \text {. }
$$

i.e.

$$
p=\frac{1}{1+G}
$$

$$
\frac{1-p}{p}=G \text {. }
$$


Equation 19 is identical to equation 6 for the heterozygous female model. Equation 20 shows that in a dioecious population with heterozygous males, the ratio of males to females is equal to the ratio of success of male- and female-determining pollen nuclei. There is no such simple proportionality in gynodioecious populations for either differential survival or gamete selection.

The rates of approach to equilibrium sex frequencies from non-equilibrium positions have been calculated from equations 15 and 17 for several situations when differential survival and gamete selection are absent and the flowers are visited only once (table 5B). As in the heterozygous female model, dioecious populations displaced from equilibrium to any value of $p$ above zero return to equilibrium in a single generation. In gynodioecious populations, the return to equilibrium is incomplete in a single generation, but is generally more rapid than with the heterozygous female model. In most instances calculated, the population returns more than 90 per cent of the displaced distance in a single generation. And with heterozygous males, unlike the heterozygous female model, sexual dimorphism is rapidly regenerated in a gynodioecious population if the females are completely lost (table 5B).

\section{Cytoplasmic inheritance model}

The rates of change and equilibrium sex proportions in a population in which the female phenotype is due to a cytoplasmic factor are calculated here by the method used for the previous model. All progeny are assumed to be the same sex as their seed parent. Then, if each flower is visited one or more times,

$$
\begin{aligned}
\text { Total seed set } & =(q \times \hat{\jmath})+(\hat{\jmath} \times \hat{\jmath}) \\
& =p\left(1-p^{x}\right)+F(1-p) .
\end{aligned}
$$

Composition of offspring

$$
=p\left(1-p^{x}\right)+Q+S F(1-p) \hat{0} .
$$

Frequency of female offspring

$$
\begin{aligned}
& =\frac{p\left(1-p^{x}\right)}{p\left(1-p^{x}\right)+S F(1-p)} \\
\Delta p & =\text { (frequency of } q \text { offspring })-p \\
& =\frac{p\left[\left(1-p^{x}\right)-p\left(1-p^{x}\right)-S F(1-p)\right]}{p\left(1-p^{x}\right)+S F(1-p)} .
\end{aligned}
$$

At equilibrium, $\Delta p=0$, i.e. $p=0$ or $1-p=0$ or $p^{x}=1-S F$

If only a fraction of female flowers are visited,

Composition of offspring

$$
=x^{\prime} p(1-p) \text { 우 }+S F(1-p) \sigma^{*}
$$

Frequency of female offspring

$$
\begin{gathered}
=\frac{x^{\prime} p}{x^{\prime} p+S F} \\
\Delta p=\frac{x^{\prime} p-p\left(x^{\prime} p+S F\right)}{x^{\prime} p+S F} .
\end{gathered}
$$


At equilibrium, $\Delta p=0$, i.e. either $p=0$ or

i.e.

$$
x^{\prime}-\left(x^{\prime} p+S F\right)=0
$$

$$
p=\frac{x^{\prime}-S F}{x^{\prime}} \text {. }
$$

The equilibrium sex proportions obtained by solution of equations 22 and 24 (table 4, figs. 6 and 7) are markedly different from those for the genic inheritance models. The frequencies pertaining in the absence of differential mortality will be considered first. Then, if the male and female seed fecundities are equal $(F=1)$, no females exist at equilibrium, except when the probabilities of fertilisation of ovules on male and female plants are equal.

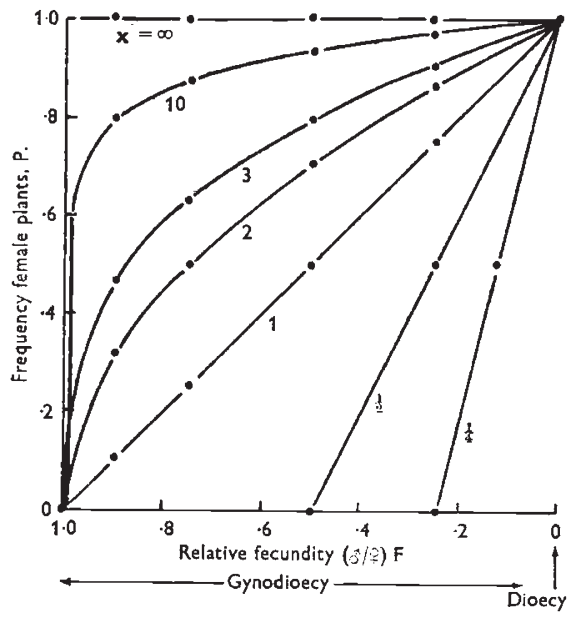

Frg. 6

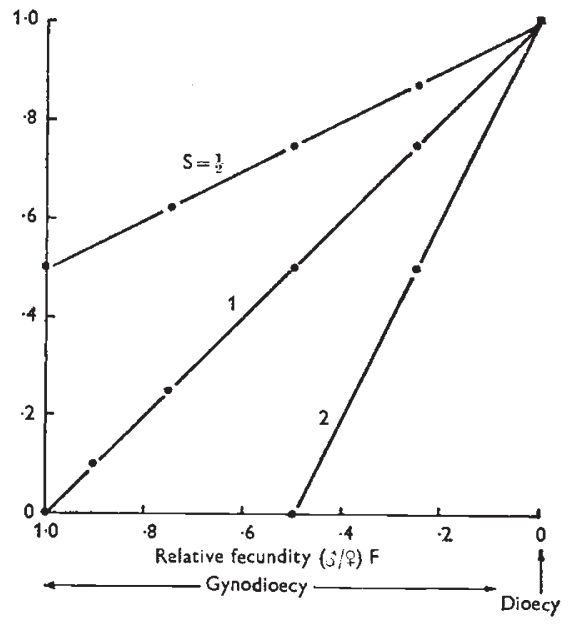

Frg. 7

Figs. 6 and 7.-Cytoplasmic model-relationships between the relative seed fecundity, $F$, and the equilibrium frequency of female plants, $p$, with various numbers of pollinator visits, $x$, and values of relative survival, $S$. Fig. 6.-Female frequency curves for different numbers of pollinator visits (shown beside each curve), when there is no differential survival $(S=1)$. Fig. 7.-Female frequency curves for different values of relative survival (shown beside each curve), when there is one pollinator visit to each flower $(x=1)$.

In the latter case, all proportions of the sexes are equally stable. At values of $F$ between 1 and 0 , females persist providing each flower is visited at least once. If only a fraction of flowers is visited, there is a maximum value of $F$, depending on the pollination level, beyond which females are eliminated (fig. 6). The female frequency increases considerably as the pollination level increases, at all values of $F$ between 1 and 0 . If the ovules of males and females are fertilised equally frequently, the population theoretically consists entirely of females, and would consequently fail to reproduce sexually. Thus unlimited pollinator activity or self-incompatibility of males in a gynodioecious population with cytoplasmically controlled sex expression would lead to extinction of a population or reliance on vegetative reproduction.

Differences in the survival rates of cytoplasmically inherited sexes have an effect on sex proportions similar to but generally larger than the effects 


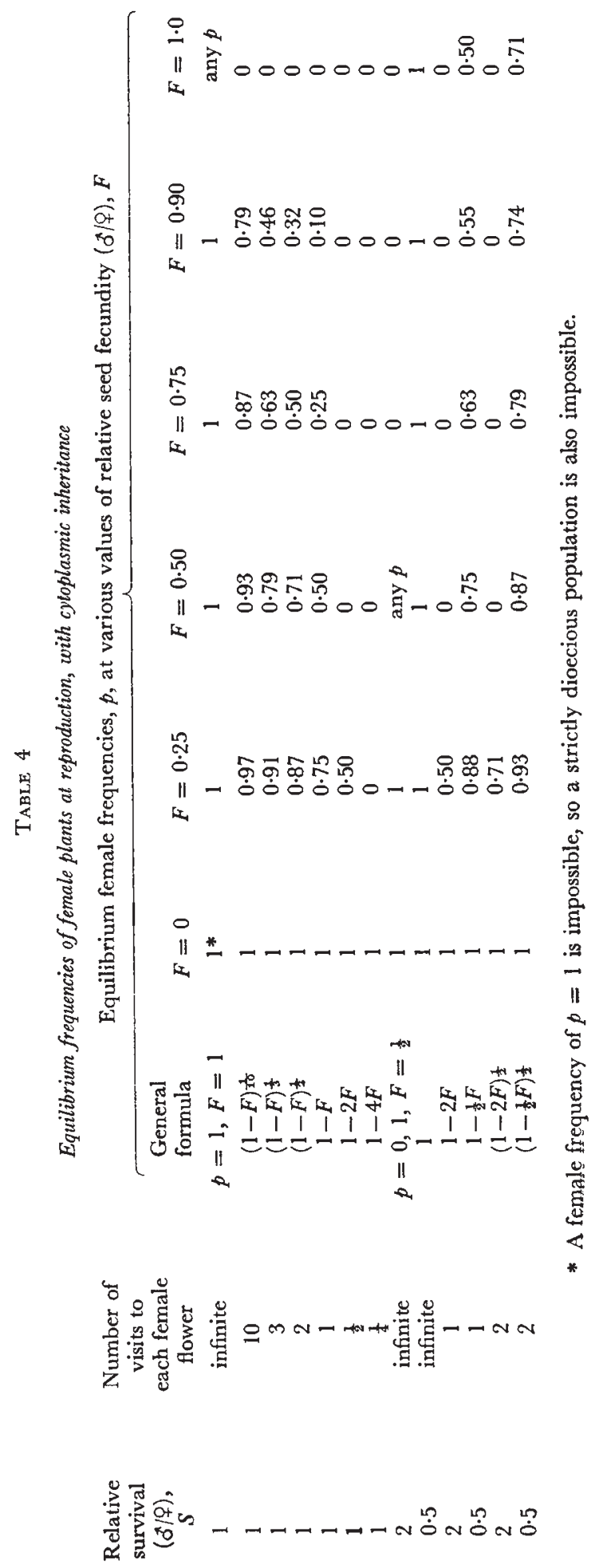


on the genic models. If the females survive at a higher rate $(S<1)$, they persist even in a population in which the male and female fecundities are equal (table 4, fig. 7). Conversely, if males survive better, the relative seed fecundity must be reduced considerably below one for females to exist at equilibrium, even if the number of pollinator visits is high.

As the male seed fecundity approaches zero, the proportion of females approaches one, for all values of relative survival and pollination levels. A

\section{Table 5}

Representative changes in female frequency in one generation from non-equilibrium frequencies, with different genetic models (no sex difference in survival, all female flowers visited once)

Genetic model and general formula for change in female frequency, $\Delta p$

A. 우 $=M m, \delta=m m$ $\Delta p=\frac{p(1-2 p-2 F)}{2 p+2 F}$
Chosen value of relative

Eq. frequency of females for value of $F$
Chosen nomequilibrium female frequency

$\begin{array}{ll}0 & 0 \cdot 500 \\ 0.25 & 0 \cdot 250 \\ 0 \cdot 25 & 0 \cdot 250 \\ 0 \cdot 25 & 0 \cdot 025 \\ 0 \cdot 50 & 0 \\ 0 \cdot 50 & 0 \\ 1.0 & 0 \\ 1 \cdot 0 & 0\end{array}$

B. $ᄋ=m m, \sigma^{\star}=M m$

$\Delta p=\frac{-4 p^{2}+p(2-3 F)+F}{4 p+3 F}$

\section{0}

$0 \cdot 25$

0.50

$1 \cdot 0$

$0 \cdot 500$

0.453

0.453

0.453

$0 \cdot 422$

$0 \cdot 390$

\section{Cytoplasmic} inheritance

$\Delta p=\frac{p(1-p-F)}{p+F}$

$\begin{array}{ll}0 & 1 \cdot 0 \dagger \\ 0.25 & 0.750 \\ 0 \cdot 25 & 0 \cdot 750 \\ 0.50 & 0.500 \\ 1 \cdot 0 & 0 \\ 1 \cdot 0 & 0\end{array}$

$p$
$0 \cdot 30$
$0 \cdot 20$
0
$0 \cdot 10$
$0 \cdot 01$
$0 \cdot 10$
$0 \cdot 01$

$\quad p$
$0 \cdot 50$
$0 \cdot 40$
0
$0 \cdot 50$
$0 \cdot 50$

Change in
in one
generation,
$\Delta p$

$0.50-p$
-0.027
+0.022
0
-0.017
-0.0002
-0.055
-0.005

$0.50-p$
-0.045
+0.047
+0.333
-0.071
-0.10

From tables 1, 3, and 4 .

$\begin{array}{lcc}-\overline{0.70} & +\overline{0.050} & -\overline{0.037} \\ 0 & +0.750 & 0 \\ 0.60 & -0.100 & -0.054 \\ 0.10 & -0.100 & -0.009 \\ 0.01 & -0.010 & -0.0001\end{array}$

$\dagger$ A female frequency of $p=1$ is impossible (see text).

population in which sex is cytoplasmically inherited and in which the male seed fecundity is low would, therefore, be expected to have a majority of females under all conditions. A strictly dioecious, cytoplasmically controlled population is impossible, since males would disappear.

The rate of change in the proportion of females in populations displaced from equilibrium has been calculated from equation 21 (or 23) for the case when flowers are visited only once and the sexes survive equally well (table 5C). The rate of approach towards equilibrium is intermediate between the corresponding rates for the heterozygous male and heterozygous female models. In the extreme case when females are entirely lost, however, the cytoplasmic model resembles the heterozygous female model in that females are not regained except by mutation or gene flow. 


\section{Discussion}

The equilibrium female frequencies derived above for the situations in which there is no differential mortality or selective fertilisation among the sex genotypes agree generally with previous results of Lewis (1941) and Ross and Shaw (1971). In particular, the heterozygous female and dual male models require the seed contribution of males to be less than half that of females if the females are to persist in the population, and the sexes approach equality in numbers as the male seed contribution falls to zero. With the cytoplasmic model, females persist if the male fecundity is only slightly less than that of females and the sex ratio approaches an all-female population as the male fecundity moves towards zero. But the introduction above of a precise model for pollination levels and of the extra parameters, differential survival and selective fertilisation, show that the behaviour of both genic and cytoplasmic models is complex; under some conditions, the conclusions of Lewis (1941) and Ross and Shaw (1971) no longer hold. In addition, in the heterozygous male model females persist at considerable frequency even if the male and female fecundities are equal, further reducing the distinction between genic and cytoplasmic modes of inheritance which Lewis (loc. cit.) stressed.

Despite the differences between genetic models, the three parameterspollination level, relative seed fecundity and relative survival-affect all the models studied above in the same general way. The proportion of females is in all cases increased by either a decrease in the seed set of males, or an increase in pollinator activity, or a decrease in the survival of males compared with females. The sensitivity of the various genetic models towards changes in the controlling parameters, however, varies considerably. In general, variation in any of the parameters causes the greatest change in equilibrium sex proportions in the cytoplasmic model, has an intermediate effect on the dual male and heterozygous female models and has the least effect on the heterozygous male model. On the other hand, selective fertilisation can occur only in the dual male model, where it has not yet been studied, and the heterozygous male model, where its effect is similar to that of differential survival.

One way in which sexual dimorphism may evolve is by the establishment of females in a previously hermaphrodite population. If the females persist, the "hermaphrodites" necessarily contribute more genes via pollen than via ovules (Lloyd, unpublished) and, on the conventions adopted herein, thereby become males in a gynodioecious population. The models examined above demonstrate the general conditions necessary for females to persist. It is possible that a male-sterile mutant might be favoured by a higher survival rate (or if it is determined by a dominant allele, by selective fertilisation), that is $S$ or $G<1$. But there are no general reasons why this should be so, and it is particularly difficult to explain how the two-fold advantage necessary for the establishment of gene-controlled gynodioecy* might arise by differential survival or selective fertilisation. On the other

* With the heterozygous male model, females need not be more than twice as fecund as males to persist in a population. But where this model applies to gynodioecious populations, as in some Carica papaya cultivars, these are probably more likely to be derived by artificial selection from dioecious ancestors with inviable YY genotypes than from hermaphrodite ancestors. 
hand, the seed-contribution of a male-sterile mutant might be immediately boosted well beyond that of the original hermaphrodites by an increased heterosis accruing from the enforced outbreeding of male-sterile plants, i.e. $F \ll 1$. The seed production of male-sterile mutants may also be augmented by the diversion of resources used for producing pollen in hermaphrodites. Hence gynodioecy may arise because of the greater number or quality of the seeds of female plants. On this view, it is not necessary to postulate that the increased outbreeding associated with gynodioecy confers any advantage on the population as a whole through increased genetic recombination and evolutionary potential.

In genera with long-established gynodioecious systems, the seed fecundity of males is usually lower than that of females not only because of the higher fitness of seed borne on females, but also because many flowers of males set little or no seed (e.g. Burrows, 1960; McCusker, 1962; Connor, 1965a). Apparently, the establishment of females to produce a gynodioecious population is frequently followed by a reduction in the seed set on males. The relative fecundity values in gynodioecious species will be considered more fully elsewhere. It may be noted, however, that there is no obvious break between the range of values in gynodioecious populations and dioecy $(F=0)$. Löve (1957), Carlquist (1966), Ross (1970) and Lloyd (1973a) have recently suggested that in some instances gynodioecy may have evolved to dioecy by reduction of the male seed contribution to zero. The possibility and consequences of the evolution of dioecy from gvnodioecy depend on the genetic model.

The equilibrium sex proportions obtained for the models examined above and those considered by Lewis (1941) and Ross and Shaw (1971) show that there is no theoretical objection to the evolution of dioecy with any of the systems controlled by one or two nuclear genes. All genic models predict a smooth transition as male fecundity is reduced to equal abundance of the sexes in dioecious populations (in the absence of differential mortality or selective fertilisation). With the heterozygous female and heterozygous male models, total loss of male seeds leads to dioecy with female and male heterogamety, respectively. The dual male model leads to dioecy with equal number of $\mathrm{Mm}$ (or XY) males and $\mathrm{mm}$ (or XX) females; that is, a conventional system of male heterogamety with no $M M(\mathrm{YY})$ individuals, although they are fully viable and fertile.

The three digenic models studied by Ross and Shaw (1971) give theoretical $\mathrm{F}_{2}$ segregation ratios of hermaphrodites (males): male-steriles (females) of $15: 1,13: 3$ and $9: 7$, respectively. The first and second of these models are basically the systems found in Plantago lanceolata (Ross, 1969) and Origanum vulgare (Lewis and Crowe, 1956) respectively, although there are further complications in both species. The sex proportions obtained with all three digenic models are identical to those obtained above for the heterozygous female and dual male models, with the same values of the parameters. In particular, when the males contribute almost no seeds, the frequencies of the sexes approach equality (Ross and Shaw, 1971). In all three models, the female genotypes are homozygous recessives at one or both loci and some of the male genotypes are heterozygous at these loci. It seems likely therefore that if dioecy evolved from any of the digenic models for gynodioecy it would be a system of simple male heterogamety with each sex present as only one genotype, as in dioecious populations derived from the dual male model. 
But the frequencies of the separate male and female genotypes needed to check this conclusion have not yet been calculated.

Evolution to strict dioecy is impossible with cytoplasmic inheritance, since it would result in extinction of the population. Moreover, if the males contribute only a small quantity of seed, the calculations performed above show that females would rapidly become predominant, even if the number of pollinator visits is low and males survive better than females. So cytoplasmic inheritance is unlikely in a gynodioecious species combining low male fecundity with male predominance or equality of the sexes. This reinforces the opinion of several authors (e.g. East, 1934; Williams, 1964) that earlier reports of cytoplasmic inheritance of gynodioecy, such as those of Correns (1928) for Satureia hortensis and Cirsium oleraceum, need reexamination.

The explanation of sex ratios in dioecious Angiosperms is relatively simple compared with that of gynodioecious species. In the absence of differential survival and selective fertilisation, equality of the sexes is expected with all genic models, at all pollination levels. The effects of differential survival are identical in dioecious species with male and female heterogamety and are also identical to the effects of selective fertilisation in the heterozygous male model; the ratio of the sexes is simply proportional to the postulated ratio of success of the gametes or zygotes. A number of examples of both male and female predominance are known in Angiosperms (Lewis, 1942; Godley, 1964). At present, male-predominant ratios have been explained primarily in terms of sex-differential survival and female-predominant ratios have been explained in terms of gamete selection (reviewed respectively in Lloyd, $1973 a$ and $1973 b)$.

The various genetic models of sexual dimorphism presented above differ in their ability to return the sex proportions to equilibrium following displacement, particularly in the extreme case of the accidental loss of females. (The loss of males results in a failure to reproduce sexually in all dioecious and gynodioecious populations, regardless of the mode of inheritance.) In populations in which sexual dimorphism is determined by the heterozygous female or cytoplasmic models, sexual reproduction among males does not produce any females. Loss of females therefore leads to a return to sexual monomorphism or extinction. On the other hand, with the heterozygous male model cross-fertilisation of any two males or self-fertilisation of a single male can rapidly regenerate sexual dimorphism following the loss of females. Similarly, with all three digenic modes of inheritance considered by Ross and Shaw (1971), both male and female offspring are produced following crosses between a proportion of the male genotypes or by self-fertilisation of those male genotypes which are heterozygous at one or both loci.

The occasional appearance in gynodioecious species of populations lacking females (e.g. Ross, 1969) demonstrates that females are in fact sometimes lost from gynodioecious populations. Moreover, sexual dimorphism in general has a scattered distribution in the Angiosperms, being characteristically restricted to small groups of species or related genera. A distribution of this nature suggests that sexual dimorphism has been frequently evolved and lost again within a relatively short evolutionary time span (Lewis, 1941; Williams, 1964). It is therefore worthwhile examining the frequency of occurrence of the various modes of inheritance in relation to their ability to regenerate sexual dimorphism following the loss of females. 
In most gynodioecious species for which the pattern of nuclear inheritance is partly or fully known, some or all males are heterozygous for the sex loci and segregate both sexes on inbreeding or interbreeding. The digenic systems discovered in Origanum vulgare (Lewis and Crowe, 1956) and Plantago lanceolata (Ross, 1969) are of this nature. The dual male model of inheritance controls gynodioecy in Cortaderia richardii (Connor, 1965b) and Mentha cunninghamii (E. J. Godley, personal communication). Some at least of the modern cultivars of Carica papaja are gynodioecious on the definitions adopted here and have the heterozygous male model of inheritance (Storey, 1953). Allen (1940) discusses a further eight gynodioecious species which produce both males and females following selfing or crossing of some or all males, but the inheritance of sex in these species is not fully known. On the other hand, the heterozygous female mode of inheritance, in which male $\times$ male offspring are all males, is known for relatively few gynodioecious species-Salvia nemorosa (Linnert, 1958), Fuchsia procumbens (Godley, 1963*), $F$. excorticata (Godley, personal communication) and Limnanthes douglasii var. rosea (Baker, 1966).

Among dioecious species there is a parallel predominance of male heterogamety. Westergaard (1958) listed 13 species or species groups (including Carica papaya) in which the heterogametic sex is reliably established. Only one of these, Fragraria, has female heterogamety. Grewal and Ellis (1972) have recently reported female heterogamety in Potentilla fruticosa L. sensu lato, also in the Rosaceae, subfamily Rosoideae. The present author has discovered a third species group, in Cotula section Leptinella (Compositae), where the female is the heterogametic sex (Lloyd, in preparation).

The predominance among both gynodioecious and dioecious species of systems of inheritance involving heterozygous males may be due to the ability of such systems to regenerate sexual dimorphism when females are lost, rather than to the comparative frequency of evolution of these systems. The ability of gynodioecious systems with heterozygous males to regenerate females is obvious, since the males characteristically reproduce to some extent via seed production. Systems with heterozygous males may have survived more frequently in the ancestors of extant dioecious species as well, in two ways. First, the males and females of dioecious species are rarely absolutely unisexual, although inconstancy of the sexes does not normally play a significant role in the breeding system, by definition. In many dioecious species, males are less strictly constant than females (e.g. Allen, 1940; Smith, 1963). Second, if dioecy has sometimes evolved via gynodioecy as postulated above, the more frequent survival of gynodioecious ancestors with heterozygous males than of ancestors with heterozygous females may have contributed to the prevalence of male heterogamety among dioecious species.

An alternative hypothesis to explain the prevalence of male heterogamety in dioecious Angiosperms has been proposed by Mulcahy (1967b). He showed that in Silene alba, which has a predominance of females in natural populations, the highest total seed set in experimental populations occurs with even more numerous females than in natural populations (Mulcahy, 1967a). He suggested that the female predominance in Silene alba is of

* Godley described $F$. procumbens as trioecious; according to the definitions adopted here, it is probably gynodioecious. 
positive selective advantage to the species because it increases the natural seed set of the total population. The cause of the unequal sex ratios in Silene alba is selective fertilisation favouring female-determining pollen over male-determining pollen (Correns, 1928). As selective fertilisation can occur only if the male is the heterogametic sex, Mulcahy (1967b) postulated that male heterogamety also is of positive selective advantage to Silene alba and possibly to other species, and that this may explain the prevalence of male heterogamety. In an accompanying paper (Lloyd, 1973b), I have suggested that selective fertilisation in Silene alba and the few other species in which it is known is better explained by the properties of the sex chromosomes in these species than by selection for maximum seed production. The objections raised there to the hypothesis that sex ratios are selected for increased seed production also apply to the additional hypothesis that this confers a selective advantage on male heterogamety.

Acknowledgments.-I am grateful to Dr M. D. Ross and Dr Margot B. Forde for critical comments on a draft of the manuscript.

\section{REFERENCES}

Allen, C. E. 1940. The genotypic basis of sex-expression in Angiosperms. Biol. Rev., 6, 227-300.

BAKER, H. G. 1966. The evolution of floral heteromorphism and gynodioecism in Silene maritima. Heredity, 21, 689-692.

Burrows, c. J. 1960. Studies in Pimelea. I. The breeding system. Trans. Roy. Soc. New Zeal., 88, 29-45.

CARLQUIST, c. 1966. The biota of long-distance dispersal. IV. Genetic systems in the floras of oceanic islands. Evolution, 20, 433-455.

CONNOR, H. E. 1965a. Breeding systems in New Zealand grasses. V. Naturalised species of Cortaderia. New Zeal. Jour. Bot., 3, 17-23.

CONNOR, H. E. 1965b. Breeding systems in New Zealand grasses. VI. Control of gynodioecism in Cortaderia richardii (Endl.) Zotov. New Zeal. Jour. Bot., 3, 233-242.

CORrens, c. 1928. Bestimmung, Vererbung und Verteilung des Geschlechtes bei den höheren Pflanzen. Handb. Vererbungsw., 2, 1-138.

EAst, е. м. 1934. The nucleus-plasma problem II. Amer. Nat., 68, 402-439.

GODLEY, E. J. 1963. Breeding systems in New Zealand plants. 2. Genetics of the sex forms in Fuschia procumbens. New Zeal. Jour. Bot., 1, 48-52.

GoDley, E. J. 1964. Breeding systems in New Zealand plants. 3. Sex ratios in some natural populations. New Zeal. Four. Bot., 2, 205-212.

Grewal, M. S., AND ellis, J. R. 1972. Sex determination in Potentilla fruticosa. Heredity, 29, 359-362.

JAIN, s. K. 1961. On the possible adaptive significance of male sterility in predominantly inbreeding populations. Genetics, 46, 1237-1240.

LEwIS, D. 1941. Male sterility in natural populations of hermaphrodite plants. New Phytol., 40, 56-63.

LEWIS, D. 1942. The evolution of sex in flowering plants. Biol. Rev., 17, 46-67.

LEWIS, D., AND CROWE, L. K. 1956. The genetics and evolution of gynodioecy. Evolution, 10, 115-125.

LINNert, G. 1958. Kernesteuerte Gynodiözie bei Salvia memorosa. Zeit. indukt. Abst. Vererb., 89, 36-51.

Lloyd, D. G. 1972. Breeding systems in Cotula L. (Compositae, Anthemideae). I. The array of monoclinous and diclinous systems. New Phytol., 71, 1181-1194.

Lloyd, D. G. 1973a. Sex ratios in sexually dimorphic Umbelliferae. Heredity, 31.

Lloyd, D. G. 1973b. Female-predominant sex ratios in Angiosperms. Heredity, 31, 35-44.

Löve, A. 1957. Sex determination in Rumex. Proc. Genet. Soc. Canada, 2, 31-36.

mCCUSker, A. 1962. Gynodioecism in Leucopogon melaleucoides A. Cunn. Proc. Linn. Soc. New South Wales, 87, 286-289.

$32 / 1-0$ 
mULCAHy, D. L. 1967a. Optimal sex ratio in Silene alba. Heredity, 22, 411-423.

MULCAHY, D. L. 1967b. The selective advantage of staminate heterogamety. Taxon, 16, 280-283.

Ross, M. D. 1969. Digenic inheritance of male sterility in Plantago lanceolata. Can. Four. Genet. Cytol., 11, 729-744.

Ross, M. D. 1970. Evolution of dioecy from gynodioecy. Evolution, 24, 827-828.

ROSs, M. D., AND SHAW, R. F. 1971. Maintenance of male sterility in plant populations. Heredity, 26, 1-8.

STOREY, w. B. 1953. Genetics of the papaya. Four. Hered., 44, 70-78.

sмrтн, в. w. 1963. The mechanism of sex determination in Rumex hastatulus. Genetics, 48, 1265-1288.

valdeyron, G. 1967. Sur le système génétique du figuier, Ficus carica L.: essai d'interprétation evolutive. Ann. Inst. Nat. Agron., 5, 1-167.

WESTERGAARD, M. 1958. The mechanism of sex determination in dioecious flowering plants. Adv. Genet., 9, 217-281.

williams, w. 1964. Genetical principles and plant breeding. Blackwell, Oxford. 